\title{
Towards a 4E perspective curricula for health and medical education
}

\author{
Stefanella Costa-Cordella ${ }^{\mathrm{a}, \mathrm{b}, \mathrm{c}}$, Eilis Reardon ${ }^{\mathrm{a}, \mathrm{d}}$ \& Francisco J. Parada ${ }^{\mathrm{a}, *}$
}

\begin{abstract}
Affiliations:
a Centro de Estudios en Neurociencia Humana y Neuropsicología, Facultad de Psicología, Universidad Diego Portales. Santiago, Chile

${ }^{\mathrm{b}}$ Centro de Estudios en Psicología Clínica y Psicoterapia, Facultad de Psicología, Universidad Diego Portales. Santiago, Chile

${ }^{\mathrm{c}}$ Instituto Milenio Depresión y Personalidad (MIDAP). Santiago, Chile

d Neuroscience \& Hispanic Studies, Connecticut College. New London, CT, United States of America
\end{abstract}

\author{
${ }^{*}$ Corresponding author: \\ Francisco J. Parada, Ph.D., \\ Centro de Estudios en Neurociencia Humana y Neuropsicología, Facultad de Psicología, \\ Universidad Diego Portales, Vergara 275, Postal code: 8370076, Santiago, Chile \\ E-mail: francisco.parada@udp.cl \\ Phone: +56226762578
}

Running title: 4E perspective curricula for healthcare

Conflict of interest statement: The authors declare the research was conducted in the absence of any commercial or financial relationships that could be construed as a potential conflict of interest.

Acknowledgments: FJP thanks Dr. Palacios-García and Dra. Rossi for informative and inspiring conversations during the writing of this article, as well as Pascal and Gael for never-ending comprehension and support while writing the present article. 
Author contributions: ER and FJP developed the main concept in the context of ER's international internship at the Center for Human Neuroscience \& Neuropsychology, Universidad Diego Portales, Santiago, Chile. SCC, ER, and FJP wrote and edited the final manuscript version for publication.

Funding sources: FJP receives funding from Agencia Nacional de Investigación y Desarrollo (ANID, Chile), FONDECYT iniciación en investigación № 11180620 and FONDECYT regular № 1190610. SCC receives funding from Agencia Nacional de Investigación y Desarrollo (ANID, Chile), FONDECYT postdoctoral No XXXXX. Funding sources had no influence on the development of the present manuscript. 


\begin{abstract}
Most of the current healthcare approaches used and taught in health professional education are labeled as biopsychosocial in opposition to the former dominant biomedical model that has been largely criticized for its exclusion of psychosocial aspects of health. However, the biopsychosocial model has significant limitations.

The Embodied, Extended, Embedded and Enactive (4E) perspective offers a transdisciplinary approach to patient care, providing a robust and holistic theoretical framework that can be taught and applied in clinical practice. Moreover, the $4 \mathrm{E}$ perspective serves as an ethical, ontological, epistemological, and methodological ground for teaching, researching, and implementing future curricula, effectively integrating psychological, health, and social sciences with the humanities.

In this Eye-Opener article, we first review the biopsychosocial model and its main caveats. We then present the $4 \mathrm{E}$ perspective, focusing on some of its many implications for healthcare education and clinical practice: specifically, the possibilities of creating novel clinical hypotheses, improving treatment decisions and communication. Finally, we discuss how the $4 \mathrm{E}$ perspective opens a transdisciplinary future for healthcare and organically fit, support, and advance current integrative efforts such as the One-Health/One-World approach.
\end{abstract}

Keywords: 4E Cognition, Health Curricula, Medical Education, Healthcare 


\section{Introduction}

People from diverse backgrounds decide to pursue a career in healthcare. Impacting other people's lives, promoting well-being, and becoming central community actors are fundamental driving forces for pursuing a degree in healthcare professions [1]. However, providing appropriate healthcare heavily depends on the context in which healthcare providers interact with people seeking advice and/or treatment. Thus, perspectives in approaching patient care have varied according to the context and over time.

The traditional approach dominating the field for decades, known as the biomedical model, is primarily focused on diagnosing and alleviating symptoms based on biological variables while excluding other aspects of health (e.g., psychosocial). The biomedical model has been extensively considered reductionist, unable to explain the complex nature of health conditions $[2,3]$. The biomedical model's mind-body dualism is evident in its application in clinical practice, where the healthcare provider's role is limited to looking at the apparent biological facts behind a person's discomfort. Fortunately, most current healthcare approaches are labeled as biopsychosocial (BPS) $[2,3]$. Even though over forty years of scholarship supports the BPS model [4], the model is still poorly defined, making it difficult to teach and apply. Reviewing both biomedical and BPS models in more detail exceeds the scope of this manuscript [5].

In the present Eye-Opener article, we start by briefly reviewing the BPS model, we then introduce the 4E perspective [6] that has the potential to build on the integrational effort already established by the BPS model. The $4 \mathrm{E}$ perspective is a robust and holistic theoretical approach that can be easily integrated into curricula, as well as taught and applied into clinical practice. It furthermore affords a transdisciplinary approach to patient care as well as a change in the very conceptualization of health and disease states. Finally, we briefly discuss four of the main implications for future research and development of the $4 \mathrm{E}$ perspective on health and medical education.

\section{Revisiting the BioPsychoSocial model}

The biopsychosocial (BPS) model was designed to be much more holistic than the traditional biomedical model. The BPS model considers that no phenomenon can exist in isolation and that biological, psychological, and social interacting factors are at the core of a patient's health. For example, the COVID-19 pandemic has led to many people being socially isolated [7]. We know that social isolation is similar to having high blood pressure or being overweight, meaning it can be as severe for someone's health as these non-communicable conditions [8,9]. Therefore, knowing if 
a person is experiencing social isolation can help physicians understand the source of the problem and better offer advice or treatment.

Another example is chronic stress, which has been shown to lead to inflammation and therefore leads to various life-threatening health states [10,11], including cancer [12] and depression [13]. Trying to understand patients as unique, contextualized individuals is precisely why the BPS model rapidly replaced the traditional biomedical view. The BPS conceptualization of a patient allows for a more thorough diagnosis because practitioners will consider different social and psychological determinants of health rather than just biological facts. However, after over forty years of scholarship, the BPS model has not been integrated into everyday practice, and its critical assessment continues $[5,14,15]$.

First, the BPS model lacks real integration of its three dimensions. Ultimately, this means only one or two aspects are -at most- considered at a time. Hence, the BPS model lives in the theory, while reductionist approaches are still the beating heart of the practice. It is important to mention that reductionist models can nevertheless provide valuable insights for practice and research. For example, a neuro-reductionist model that considers cognition as ontologically happening in the brain will search and treat brain-related malfunctions. Leading to novel discoveries and further understanding of the studied systems. However, reductionist models fall short when faced with the complexity of the human experience of health and disease.

Second, critics have pointed out that -even if the three BPS dimensions are considered- no actual links between the implemented dimensions exist. Hence, each dimension is a separate world. This is reminiscent of mind/body dualism, which replicates in neuroscience and other disciplines [16]. For the BPS model to succeed, the three different components need to be linked together and conceptualized as integrated dimensions rather than independent dominions that require to be thought of as a unity. This divide is greatly experienced by mental health students, practitioners, and patients, as mental health and physical health are rarely thought of as one and one alone [17]. Artificial hierarchies and detrimental power asymmetries within and between medical teams are the evident results of such a divide, where physical health practitioners and/or students are thought to be on top of their mental health colleagues. Hence, to avoid these and other unintended consequences, the BPS model should incorporate each of its dimensions as a single and dynamic constitutive aspect of experiencing health and disease states.

Finally, the third criticism concerns, precisely, the human experience of health and disease. Even if the BPS model manages to integrate its dimensions successfully, it never really addresses how the patient is feeling in the moment and what they are experiencing during the disease states. In the realm of mental health, this might be less true since talking about one's experience is crucial to 
many aspects of mental health treatments (e.g., psychotherapy [18], art therapy [19]). This criticism is essential since patients often feel dehumanized during visits to some practitioners, especially if they are not asked about how they are feeling or what they are actually experiencing at that moment. The following section will review three principles and a suggested conclusion encompassed within the "4E perspective" concept.

\section{The Embodied, Extended, Embedded and Enactive (4E) perspective}

Truly integrating the underlying biological factors, the psychological processes, and the social determinants of health and disease states is impossible without understanding the constitutive components of human experience [20]. We think that the first step to reach such integration is to accept the three following non-trivial principles: First, experience is a biological phenomenon (i.e., Embodiment). It is embodied in all physiological processes, neural, endocrine, circulatory, and otherwise [21]. No dichotomies between body and mind are therefore needed. Physical health is mental health and viceversa; our curricula, future practice, and public policy should reflect that.

Second, experience incorporates the world (i.e., Extendedness). Through each life's trajectory (i.e., ontogeny) experience is dynamically extended into the material/virtual, technological, sociocultural, sociopolitical, demographic, and geographic environments which afford it [22]. Those dynamic and functional body/world couplings scaffold human experience, enriching or impoverishing it [20]. The use of devices, technologies, and other assistance/enhancement strategies for augmenting and/or regulating functioning (e.g., physical, cognitive, emotional) is therefore natural and should be normalized and promoted. Our curricula, future practice, and public policy should be oriented towards eliminating sources of stigma and inequalities regarding access and/or usage of such assistance/enhancement strategies and devices.

Third, experience happens in the world (i.e., Embeddedness). It is embedded and inseparable from the material/virtual, technological, sociocultural, sociopolitical, demographic, and geographic environment which affords it [23]. Reframing individuality is therefore needed [24]. Individual, global, and planetary health/disease states all depend on each other and on complex macro-level factors; our curricula, future practice, and public policy should reflect that as well.

The ideas, theoretical needs, and future directions encompassed within these three principles suggest that more comprehensive models are needed for understanding cognition. We think the Enactive approach offers such complexity [21,25]. Thus, the ethico-onto-epistemology known as the $4 E$ perspective to Cognition or simply 4E-Cognition [6], as a truly biogenic perspective [26], offers a new material/natural way of being present in the world. It facilitates and fosters a transversal 
and holistic way of thinking; connecting students, researchers, and practitioners with "human exceptionalism while being accountable for the role we play in the differential constitution and differential positioning of the human among other creatures" (p. 136) [27].

Thus, even though the $4 \mathrm{E}$ perspective has mostly been kept in the philosophy of mind realm [6], we think ethico-onto-epistemologies such as the 4E (and other similar approaches and perspectives [e.g., 28]) may be the model that future healthcare providers need to be exposed to whilst going through their training. We think the $4 \mathrm{E}$ perspective contains all of the positive elements that are present in the BPS model, while taking into account the parts that are missing from it; minding human experience and human becoming [29]. Furthermore, it contains specific methodological tools for basic and translational research. Recently, the use of mobile unobtrusive sensor technologies provide the necessary means for the methodological research implementation of the $4 \mathrm{E}$ perspective [30].

Given the multi- and transdisciplinary nature of the 4E perspective, there are several ways to implement its principles to health and medical education, research, and practice, as well as health-oriented public policy [25,31,32]. Considering the fact that the consequences of the perspective are not trivial, and furthermore this is currently ongoing work, deeply exploring each one of these dimensions is beyond the scope of the present piece. We certainly hope to motivate this discussion among medical students, instructors, and practitioners. Nevertheless, in the following section we will outline some of the main implications of the $4 \mathrm{E}$ perspective to Health and Medical Education.

\section{Implications of the 4E perspective in Health and Medical Education}

Adopting the 4E perspective and effectively translating its principles into health education curricula and clinical practices is not straightforward: i) it implies acknowledging that experiencing health and disease happens in bodily beings, whose life trajectories are constitutively and mechanistically intertwined with their physiological states and the material, sociopolitical, and cultural contexts where those biological dynamics unfold; ii) it also implies acknowledging that experiencing health and disease will drastically change how people make sense of their world and -therefore- how people ultimately engage themselves with the physical and sociopolitical world. Considering these two global implications, we will focus on four action domains, which are transversal in medical education curricula and dynamically emerge during clinical practice. 


\section{Generating novel clinical hypotheses}

The 4E perspective invites to re-conceptualize the usefulness of current health, disease, and intervention models (e.g., BPS-based perspectives). As mentioned earlier, neuro-reductionist models will search and treat brain-related malfunctions, missing the physiological (e.g., the brain/body dynamics underlying any brain phenomena [33]) and experiential richness (e.g., the sociomaterial world [34]) where maladies unfold. More complex approaches have been recently suggested. For example, de Haan [32] and Maiese [31] independently identified mind/body dualism as one of the main weaknesses of the current Western worldview of health. Interestingly, de Haan suggests the Enactive approach as a novel model for psychiatry finally replacing both the biomedical and the BPS models, leading to better conceptualization of mental health and treatment outcomes [32]. Similarly, Maiese generates a novel hypothesis of mental health conditions, beyond the reductive BPS disease concept or the over-complexity of the socio-constructivist view. Maieses's proposal is based on the idea that mental health conditions can be thought of as disruption of sense-making processes, fracturing the person's dynamical engagement and functional body/world coupling abilities [20,31]. Implementing novel ideas, such as those proposed by de Haan and Maiese, will change our conceptualization of health and disease, thus changing the kinds of questions we ask, the kinds of clinical hypotheses we seek, and the methods and tests employed to such ends. Future medical practitioners will search and test novel hypotheses of health and disease, critically considering the complexity of each person.

\section{Optimizing treatment decisions}

The 4E perspective has many implications for how treatment decisions are made regarding patient care. As mentioned earlier, other models lead to treatment decisions being made fast and efficiently, since the goal is to treat a specific situation, and move on. However, looking through the lens of the $4 \mathrm{E}$ perspective will allow treatment decisions to be efficient due to the nature of them being comprehensive and integrated into the experience and sociomaterial reality of patients [35]. Social, psychological, cultural, physiological, and biological aspects of each person will be taken into account to determine the treatment(s), decided after considering the dimensionality of the patient. The 4E perspective aims for a holistic screening of people seeking care, improving treatment decisions as they will make sense to patients, their families, and their sociomaterial context. This also has implications for better health in the rest of patients' lifespan, since a more accurate and concrete treatment decision considering as many factors as possible will likely be better for patients' overall 
health. Future medical practitioners will better and meaningfully connect with their patients and families, co-design treatments, and consensually reach lifestyle-consistent decisions.

\section{Improving communication between patients and practitioners}

Medical practitioners are often criticized for being "cold" and/or "distant" in the office setting, not caring for the person seeking care [36]. Patients might feel that health practitioners are there to evaluate them and move on to the next case, getting the job done without really connecting or caring. However, the $4 \mathrm{E}$ perspective promotes teaching to explore the structure and richness of a patient's experience. Practitioners will benefit from asking questions about a patient's social life, psychological well being, culture, and environment. Thus, they will be more likely to engage in meaningful clinical interviews with the patients and their families, improving communication and treatment adherence. The structure and organization of healthcare systems will be of special relevance for accomplishing this. Future medical practitioners will better and meaningfully communicate with their patients and families, effectively communicating any treatments, lifestyle-consistent decisions, implications, difficulties, and benefits.

\section{Creating a transdisciplinary future}

The 4E perspective offers a change in the ontology of health and disease states, the constitutiveness of what these states really are and really mean for a person experiencing them. The plethora of factors constituting health -from chemical exposure [37] to interspecies interaction [38], public policy [39] and climate change [40]- need a framework that can make justice to such complexity [35,39]. The 4E perspective effectively foregrounds the constitutive role of the complexity of life -from biophysical to sociomaterial and interactional dimensions [21,25]- while, at the same time, considering cultural and interpersonal variability as crucial information for healthcare. Some paths might lead to events creating fluctuations in this rich tapestry, significantly affecting development, while others might be more evident in the cognitive or sensorimotor dimensions. Some fluctuations might be transient, while others might generate a shift in the path's course with great impact on the person's physiology, meanings, sensorimotor and/or cognitive skills. At the intersection of these paths is the person seeking help at a given point in time. Health practitioners' difficult -yet enormously beautiful- task is to comprehend the path-dependent rich diversity of human bodies and their unique experiences. Furthermore, healthcare practitioners must offer a novel event that will trigger a new path fluctuation -without negating or minimizing the patient's experience- but increasing her autonomy. This monumental task cannot be reached alone. 
The 4E perspective defines healthcare as a transdisciplinary effort implemented by effectively integrated transdisciplinary teams. Future health practitioners will actively engage and promote transdisciplinarity.

\section{Concluding Remarks}

The future implementation of the $4 \mathrm{E}$ perspective in healthcare education promises to further the amazing benefits both the biomedical and the BPS models have brought to society, improving human wellbeing. Importantly, the 4E perspective is compatible with modern healthcare conceptualizations, such as the One Health/One World approach [41]. One Health/One World is based on the idea that individuals' health is associated with their shared sociomaterial environment as well as other species' health. The current COVID-19 pandemic has provided all necessary evidence to strengthen and implement initiatives like the One Health/One World approach, raising the need to integrate psychological, biological, and health sciences effectively.

Importantly, other animals play various roles in human lives, whether as a source of food, company, service and/or social buffering $[42,43]$. Interspecies interactions allow for microbial transmissions, which inevitably occurs for good and bad [44,45]. More than $60 \%$ of known infectious diseases in humans and 70\% of the emerging infections are zoonotic conditions transmitted from other species to humans $[41,46]$. More importantly, the zoonotic infection can go from human to other species, meaning that other animals may act as reservoirs of diseases for future, more aggressive infections $[47,48]$, encountering suitable grounds for fast spreading in the modern globalized socioeconomic reality. These warnings apply not only to zoonotic diseases, but also to food safety [49]. The 4E perspective offers an ethico-onto-epistemology compatible with the One Health/One World approach, effectively articulating a theoretical bridge between biological, health, social sciences, and the humanities. This bridge can be implemented both as specific course material and as transversal content, at both undergraduate and graduate levels [39]. Collaboration needs to take place, which is the purpose of the One Health/One World approach, between those that study humans, animals, and the environment [50].

The complexity of the modern world requires a comprehensive approach capable of understanding and integrating the path-dependent diversity of human lives. The 4E perspective offers the ethical, ontological, epistemological, and methodological components articulating a transdisciplinary future. Promising integrative frameworks for future healthcare -such as the One Health/One World approach- and future health curricula will highly benefit from using the $4 \mathrm{E}$ perspective as the theoretical ground for effective change. 


\section{References}

1. Fox K, Corstorphine W, Frazer J, Johnstone A, Miller A, Shepherd N, et al. Ten reasons why every junior doctor should spend time working in a remote and rural hospital. Future Healthc J. ncbi.nlm.nih.gov; 2020;7:12-4.

2. Engel GL. A unified concept of health and disease. Perspect Biol Med. 1960;3:459-85.

3. Engel GL. The need for a new medical model: a challenge for biomedicine. Science. science.sciencemag.org; 1977;196:129-36.

4. Bolton D, Gillett G. The Biopsychosocial Model of Health and Disease: New Philosophical and Scientific Developments. Cham (CH): Palgrave Pivot; 2019.

5. Lane RD. Is it possible to bridge the Biopsychosocial and Biomedical models? Biopsychosoc Med. Springer; 2014;8:3.

6. Newen A, De Bruin L, Gallagher S, editors. The oxford handbook of 4E cognition. London, England: Oxford University Press; 2018.

7. Venkatesh A, Edirappuli S. Social distancing in covid-19: what are the mental health implications? BMJ. 2020. p. m1379.

8. Leigh-Hunt N, Bagguley D, Bash K, Turner V, Turnbull S, Valtorta N, et al. An overview of systematic reviews on the public health consequences of social isolation and loneliness. Public Health. Elsevier; 2017;152:157-71.

9. Cacioppo JT, Cacioppo S. Social Relationships and Health: The Toxic Effects of Perceived Social Isolation. Soc Personal Psychol Compass. Wiley Online Library; 2014;8:58-72.

10. Yaribeygi H, Panahi Y, Sahraei H, Johnston TP, Sahebkar A. The impact of stress on body function: A review. EXCLI J. ncbi.nlm.nih.gov; 2017;16:1057-72.

11. Fioranelli M, Bottaccioli AG, Bottaccioli F, Bianchi M, Rovesti M, Roccia MG. Stress and Inflammation in Coronary Artery Disease: A Review Psychoneuroendocrineimmunology-Based. Front Immunol. frontiersin.org; 2018;9:2031.

12. Dai S, Mo Y, Wang Y, Xiang B, Liao Q, Zhou M, et al. Chronic Stress Promotes Cancer Development. Front Oncol. internal-journal.frontiersin.org; 2020;10:1492.

13. Cryan JF, O'Riordan KJ, Sandhu K, Peterson V, Dinan TG. The gut microbiome in neurological disorders. Lancet Neurol. Elsevier; 2020;19:179-94.

14. Lehman BJ, David DM, Gruber JA. Rethinking the biopsychosocial model of health: Understanding health as a dynamic system. Soc Personal Psychol Compass. Wiley; 2017;11:e12328. 
15. Stilwell P, Harman K. An enactive approach to pain: beyond the biopsychosocial model. Phenomenol Cognitive Sci. Springer; 2019;18:637-65.

16. Mudrik L, Maoz U. “Me \& my brain”: exposing neuroscience’s closet dualism. J Cogn Neurosci. MIT Press - Journals; 2015;27:211-21.

17. Kolappa K, Henderson DC, Kishore SP. No physical health without mental health: lessons unlearned? Bull World Health Organ. SciELO Public Health; 2013;91:3-3A.

18. Koole SL, Tschacher W. Synchrony in Psychotherapy: A Review and an Integrative Framework for the Therapeutic Alliance. Front Psychol. frontiersin.org; 2016;7:862.

19. King JL. Art Therapy, Trauma, and Neuroscience: Theoretical and Practical Perspectives. Routledge; 2021.

20. Rojas-Líbano D, Parada FJ. Body-World Coupling, Sensorimotor Mechanisms, and the Ontogeny of Social Cognition. Front Psychol. frontiersin.org; 2019;10:3005.

21. Varela FJ, Thompson E, Rosch E. The Embodied Mind: Cognitive Science and Human Experience. MIT Press; 1991.

22. Clark A, Chalmers D. The Extended Mind. Analysis. Oxford University Press (OUP); 1998;58:7-19.

23. Kapilashrami A, Hankivsky O. Intersectionality and why it matters to global health. Lancet. thelancet.com; 2018;391:2589-91.

24. Gilbert SF, Sapp J, Tauber AI. A symbiotic view of life: we have never been individuals. Q Rev Biol. journals.uchicago.edu; 2012;87:325-41.

25. Di Paolo E, Buhrmann T, Barandiaran X. Sensorimotor Life: An Enactive Proposal. Oxford: Oxford University Press; 2017.

26. Lyon P. The biogenic approach to cognition. Cogn Process. 2006;7:11-29.

27. Barad K. Meeting the Universe Halfway. Duke University Press; 2007.

28. Zurn P, Shankar A. Curiosity Studies: A New Ecology of Knowledge. U of Minnesota Press; 2020.

29. De Jaegher H, Di Paolo E. Participatory sense-making. Phenomenol Cognitive Sci. Springer; 2007;6:485-507.

30. Parada FJ, Rossi A. Perfect timing: Mobile brain/body imaging scaffolds the 4E-cognition research program. Eur J Neurosci [Internet]. Wiley; 2020; Available from:

https://onlinelibrary.wiley.com/doi/abs/10.1111/ejn.14783

31. Maiese M. An enactivist reconceptualization of the medical model. Philos Psychol. Routledge; 2021;1-27. 
32. de Haan S. An Enactive Approach to Psychiatry. Philos Psychiatr Psychol. Johns Hopkins University Press; 2020;27:3-25.

33. Rebollo I, Tallon-Baudry C. The sensory and motor components of the cortical hierarchy are coupled to the rhythm of the stomach during rest. J Neurosci [Internet]. 2022; Available from:

http://dx.doi.org/10.1523/JNEUROSCI.1285-21.2021

34. Robinson JM, Mills JG, Breed MF. Walking Ecosystems in Microbiome-Inspired Green Infrastructure: An Ecological Perspective on Enhancing Personal and Planetary Health. Challenges. Multidisciplinary Digital Publishing Institute; 2018;9:40.

35. Robinson JM, Breed MF. Green Prescriptions and Their Co-Benefits: Integrative Strategies for Public and Environmental Health. Challenges. Multidisciplinary Digital Publishing Institute; 2019;10:9.

36. Stepien KA, Baernstein A. Educating for empathy. J Gen Intern Med. Springer; 2006;21:524-30.

37. Vermeulen R, Schymanski EL, Barabási A-L, Miller GW. The exposome and health: Where chemistry meets biology. Science [Internet]. American Association for the Advancement of Science; 2020 [cited 2021 Dec 28]; Available from: https://science.sciencemag.org/content/367/6476/392.abstract

38. Postler TS, Ghosh S. Understanding the Holobiont: How Microbial Metabolites Affect Human Health and Shape the Immune System. Cell Metab. Elsevier; 2017;26:110-30.

39. Ishaq SL, Parada FJ, Wolf PG, Bonilla CY, Carney MA, Benezra A, et al. Introducing the Microbes and Social Equity Working Group: Considering the Microbial Components of Social, Environmental, and Health Justice. mSystems. Am Soc Microbiol; 2021;6:e0047121.

40. Mendenhall E, Singer M. The global syndemic of obesity, undernutrition, and climate change. Lancet. thelancet.com; 2019. p. 741.

41. Bhatia R. Implementation framework for One Health approach. Indian J Med Res. ncbi.nlm.nih.gov; 2019;149:329-31.

42. Gorman R. Therapeutic landscapes and non-human animals: the roles and contested positions of animals within care farming assemblages. Social \& Cultural Geography. Routledge; 2017;18:315-35.

43. Haynes RP. Competing conceptions of animal welfare and their ethical implications for the treatment of non-human animals. Acta Biotheor. Springer; 2011;59:105-20.

44. Misic AM, Davis MF, Tyldsley AS, Hodkinson BP, Tolomeo P, Hu B, et al. The shared microbiota of humans and companion animals as evaluated from Staphylococcus carriage sites. Microbiome. Springer; 2015;3:2.

45. Kates AE, Jarrett O, Skarlupka JH, Sethi A, Duster M, Watson L, et al. Household Pet Ownership and the Microbial Diversity of the Human Gut Microbiota. Front Cell Infect Microbiol. frontiersin.org; 2020;10:73. 
46. Rahman MT, Sobur MA, Islam MS, Ievy S, Hossain MJ, El Zowalaty ME, et al. Zoonotic Diseases: Etiology, Impact, and Control. Microorganisms [Internet]. mdpi.com; 2020;8. Available from: http://dx.doi.org/10.3390/microorganisms8091405

47. Munir K, Ashraf S, Munir I, Khalid H, Muneer MA, Mukhtar N, et al. Zoonotic and reverse zoonotic events of SARS-CoV-2 and their impact on global health. Emerg Microbes Infect. Taylor \& Francis; 2020;9:2222-35.

48. Mazet JAK, Clifford DL, Coppolillo PB, Deolalikar AB, Erickson JD, Kazwala RR. A “One Health” Approach to Address Emerging Zoonoses: The HALI Project in Tanzania. PLoS Med. Public Library of Science; 2009;6:e1000190.

49. Walls H, Baker P, Chirwa E, Hawkins B. Food security, food safety \& healthy nutrition: are they compatible? Global Food Security. Elsevier; 2019;21:69-71.

50. Tay MZ, Poh CM, Rénia L, MacAry PA, Ng LFP. The trinity of COVID-19: immunity, inflammation and intervention. Nat Rev Immunol. nature.com; 2020;20:363-74. 